\title{
Research on Node Importance Evaluation of the Directed Weighted Complex Network
}

\author{
Wei Xia ${ }^{a}$, Xinxue Liu ${ }^{b}$, Shaofei Meng ${ }^{c}$ and Jinlong Fan ${ }^{d}$ \\ The Rocket Force University of Engineering, Shaanxi Xi'an 710025, China; \\ axiawei66@163.com, ${ }^{b}$ liyax@sina.com, 'shaofei_m@sina.com, ${ }^{\mathrm{d}} 1340081949 @ q q . c o m$
}

KEYWORDS: city network objective, importance evaluation, directed weighted, vital nodes.

ABSTRACT: Aiming at the problem is neither objective nor thorough to simulate and analyze the real-world by the non-directed weighted network model, this paper not only defined a new evaluation metric for vital node importance called Directed Weighted Node Ranks(DWNR) possessing the local and entire link characteristics, but also designed a node importance evaluation model based on the directed weighted network. Initializing and ranging the nodes of networks by using the index of in-degree, the model can improve the constringency rate and identify precision. According to the simulation example, the feasibility and validity of network's node importance evaluation by this method are verified, and the results are not only effective and accurate, but also with high efficiency.

\section{Introduction}

It's very important to identify the key node by using the complex network theory, and it can increase the robustness and survivability. Nowadays, workers can use the random model ${ }^{[1]}$, small world model ${ }^{[2]}$ and scale free BA model $^{[3]}$ to research the complex network, whereas these models probably have some faults. There are two methods to evaluate the importance of network node, some people think the critical node is prominent, and the others consider the critical node can't be destroyed. In other words, it might be very harmful if the critical node was destroyed. The representative theories are displayed by the papers[4 8]. However, when evaluate the node importance of the complex networks, these theories mainly aim at the non-directed non-weighted network that don't accord with the real world, and lack of analysis for the effect, direction and relationship between the nodes. These factors induce the identify precision of the center nodes. Therefore, according to the characters of directed weighted complex networks, this paper deeply analysis the model based on BBV from direction and weight vectors $^{[9]}$, advanced a new evaluation method-DWNR designed theory, built an evaluation model and gave the simulation.

\section{Overview of network}

Complex networks can be described by graph $G=(V, E)$, where gathers $V=\left(v_{1}, v_{2}, \cdots, v_{n}\right)$ and $E=\left(e_{1}, e_{2}, \cdots, e_{m}\right)$ are the node gather and edge gather. For each edge in gather $E$, there are two corresponding nodes in gather $V$. The nodes $v_{i}$ and $v_{j}$ are linked up to the edge $e_{k}$, recorded as $e_{k}=\left(v_{i}, v_{j}\right)$, we call the points $v_{i}$ and $v_{j}$ as the endpoints of edge $e_{k}$. In the real world, networks can be abstracted as 4 types of different networks: non-directed non-weighted network, directed non-weighted network, non-directed weighted network and directed weighted network, just showed as Fig.1

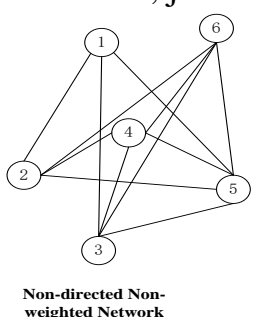

Non-directed Non-
weighted Network

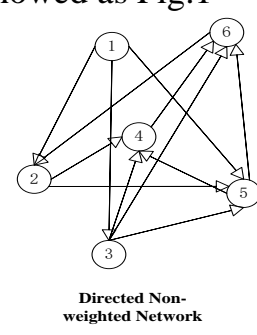

Directed Non-
weighted Network

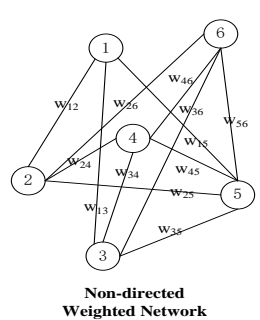

Non-directed
Weighted Network

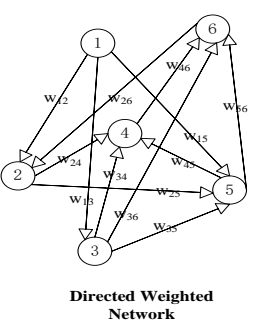

Network

Fig.1 Four types of different networks 


\section{BBV network model}

According to the structure characteristics of the complex network, combined with the direction of network evolution and characteristics, divided the node strength into in-strength and out-strength. At the same time, introduced two probability parameters $\mathrm{p}$ and $\mathrm{q}$, which can be selected in the process of evolution according to merit node strength, and by adjusting parameters $\mathrm{p}$, q to makes the model more close to the real characteristics of the network.

\subsection{Parameters description}

$w_{i j}$ : Weight of the Directed edge $\langle i, j\rangle$, and $w_{j i}$ is the weight of the Directed edge $\langle j, i\rangle$. Generally, $w_{i j} \neq w_{j i}$. $t($ ii $)$ : Node sets points to the node $i$.

$t($ io $)$ : Node sets points out from the node $i$

$S_{i i}$ : The in-strength of node $i$, the formula is $S_{i i}=\sum_{j \in t(i i)} w_{j i}$.

$S_{i o}$ : The out-strength of node $i$, the formula is $S_{i o}=\sum_{j \in t(i o)} w_{i j}$.

\subsection{Modeling process}

Step 1 Network initialization. Let the initial network is the whole coupling network having $m_{0}$ nodes, and the weight of each edge is $w_{0}$;

Step 2 Network growth. Adding style has two ways: (1)add nodes and edges at the same time. To increase a new node to the network at the probability $q$ (designated as $\left.v_{k}\right)$, the node $v_{k}$ has $m\left(m \leq m_{0}\right)$ edges, and the number of in-edges and out-edges for these new nodes obey the binomial distribution $B(m, p)$ and $B(m, 1-p)$ respectively, where $p$ satisfy the inequality $0 \leq p \leq 1$. (2) Only add edges without increasing nodes, add $m \leq m_{0}$ network edges at the probability $1-q$.

Step 3 Network preferential attachment。For both growth mode, select the node $i$ as the in-node of new edge $\left(\left\langle v_{k}, v_{i}\right\rangle\right.$ indicated that the connection from $v_{k}$ directed to $\left.v_{i}\right)$ comply with the principle of merit for the in-strength, defined as follow:

$$
\prod_{n \leftarrow i}=\frac{S_{i i}}{\sum_{j \in(\text { (ii) }} S_{j i}}
$$

Similarly, select the node $i$ as the out-node of this new edge $\left\langle\left\langle v_{i}, v_{k}\right\rangle\right.$ indicated that the connection from $v_{i}$ directed to $v_{k}$ ) comply with the principle of merit for the out-strength, defined as follow:

$$
\prod_{n \rightarrow i}=\frac{S_{i o}}{\sum_{j \in(i o)} S_{j o}}
$$

Step 4 Weight edge dynamic evolution. Each side of the initial weights are assigned as $w_{0}$, Considering at the new edge node may change the local network load and lead to changes in the weight edge of the connection point of the adjacent side occurs, adjust the rules (1) in Step 2 as follows:

a. If the new edge is the in-edge of node $i$, that's to say the edge $\left\langle v_{k}, v_{i}\right\rangle$ is from the new node $k$ to node $i$, and then adjusted the in-strength and weight of the node $i$ and its neighbor node as follows:

$$
S_{i i} \rightarrow S_{i i}+w_{0}+\delta_{i} ; \quad w_{j i} \rightarrow w_{j i}+\Delta w_{j i} ; \quad \Delta w_{j i}=\delta_{i} \frac{w_{j i}}{S_{i i}}
$$

Where $\delta_{i}$ is the additional traffic load of the node $i$.

b. If the new edge is the out-edge of node $i$, that's to say the edge $\left\langle v_{i}, v_{k}\right\rangle$ is from the new node $i$ to node $k$, and then adjusted the in-strength and weight of the node $i$ and its neighbor node as follows: 


$$
S_{i o} \rightarrow S_{i o}+w_{0}+\delta_{i} ; \quad w_{i j} \rightarrow w_{i j}+\Delta w_{i j} ; \quad \Delta w_{i j}=\delta_{i} \frac{w_{i j}}{S_{i o}}
$$

For growth style(2), there are no changes in the network at the reason of no new nodes, only the weight of the endpoints for the new edge changed dynamically, so there is with no need for considering additional load flow. If the new edge is $\left\langle v_{i}, v_{j}\right\rangle$, there are:

$$
S_{i o} \rightarrow S_{i o}+w_{0} ; \quad S_{j i} \rightarrow S_{j i}+w_{0} ; \quad w_{i j}=w_{i j}+w_{0}
$$

\section{3 parameters initialization}

Aiming at the model design for the network, initialed the network parameters on the basis of relevant literature and historical data as the following: $\delta_{i}=\delta=1.0 ; w_{0}=1.0 ; m=m_{0}=10 ; \quad p=0.5 ; q=0.3$.

\section{DWNR evaluation algorithm}

In undirected networks, the importance evaluating index of complex network nodes can be divided into two categories: node of local area connection properties indices (degree, strength, clustering coefficient) and node of global position attribute indices (characteristic path length, tightness, feature vectors). This paper defined a new measure index in the weighted network -DWNR inspired from paper[10].

\subsection{Concept of DWNR}

Suppose node $v$ has several connections just from nodes $u_{1}, u_{2}, \cdots, u_{n}$, and $w_{u v}$ is the connection strength from node $u$ to $v, \alpha \in(0,1)$ is the damping coefficient. The bigger the value of the damping coefficient is, the closer the DWNR matrix gets to a real network, but the convergence rate will be slower down, usually took $\alpha=0.85$. So, the formula of DWNR value is:

$$
N R(v)=\frac{1-\alpha}{N}+\alpha\left(\frac{w_{u_{1} v}}{\sum_{j=1}^{m_{1}} w_{u_{1} z_{j}}} N R\left(u_{1}\right)+\frac{w_{u_{2} v}}{\sum_{j=1}^{m_{2}} w_{u_{2} z_{j}}} N R\left(u_{2}\right)+\cdots+\frac{w_{u_{n} v}}{\sum_{j=1}^{m_{n}} w_{u_{n} z_{j}}} N R\left(u_{n}\right)=\frac{1-\alpha}{N}+\alpha \sum_{i=1}^{n} \frac{w_{u_{i} v}}{\sum_{j=1}^{m_{i}} w_{u_{i} z_{j}}} N R\left(u_{i}\right)\right.
$$

Where $N$ is the number of nodes in complex network; $N R(v)$ is the DWNR value of node $v ; N R\left(u_{1}\right)$ is the DWNR value of node $u_{1}$ itself pointed to node $v$.

The sum of the connection strength which sent from node $u_{1}$ is: $\sum_{j=1}^{m_{1}} w_{u_{1} z_{j}}$, and the DWNR weight of node $u_{1}$ obtained from $v$ can be showed as: ${ }^{w}$

$$
w_{u_{1} v} / \sum_{j=1}^{m_{1}} w_{u_{1} z_{j}}
$$

Obviously, the sum of the all weight sent out from node $u_{1}$ is “ 1 ”. If the value $N R(v)$ is higher the node $v$ will be more important.

\subsection{Description of DWNR}

The evaluation importance method of network in this paper Based on the idea that the importance equivalent significance, and the use the method showed in paper[11] to process the DWNR indicators.

\subsubsection{Definitions}

Definition 1 (connection matrix H ): Characterizes the relationship of the directed weighted network, the each elements in this matrix represents the strength of the connection between two nodes, and the formula is $H=\left(h_{i j}\right)_{n \times n}$, where $i 、 j$ represents the number of nodes; $n$ is the size of network, in other words, it is the number of all nodes in the network.

Definition 2 (suspended virtual node): the node $i$ with no links to the other nodes in the network, and the value of line $i$ in the connection matrix are all zeros.

Definition 3 (probability transition matrix P): obtained by normalizing the connection matrix $\mathrm{H}$, each 
element in matrix P represents a probability from one node to another. The formula is showed as: ${ }_{P=\left(\sum_{j=1} h_{i j} / h_{n \times n}\right.}$

Definition 4 (probability transition matrix $\mathrm{P}^{\prime}$ ): normalized the connection matrix $\mathrm{H}$ after finishing deal with suspended virtual nodes. That's to say, use polynomial $(1 / n) e^{T}$ replace the all zero rows in connection matrix $\mathrm{H}$, and then divided by sum of the row nonzero vector.

$$
P^{\prime}=P+\eta(1 / n) e^{T}=\left(h_{i j} / \sum_{j=1}^{n} h_{i j}+\eta \times 1 / n\right)_{n \times n}
$$

Where $n$ is the scale of the network; $\eta$ is the Boolean vector to judge suspended virtual nodes, one or zero represents the node $v_{i}$ is or is not the suspended virtual node.

Definition 5 (transposed matrix M ): obtained by dealing with the transition probability matrix $\mathrm{P}^{\prime}$, the formula is:

$$
M=P^{\prime T}=\left(h_{i j} / \sum_{j=1}^{n} h_{i j}+\eta \times 1 / n\right)_{n \times n}^{T}
$$

Define 6 (DWNR matrix A ): obtained by use the formula (6) as follows:

$$
A=\alpha \times M+(1-\alpha) \times \frac{1}{n} e e^{T}
$$

Where $\alpha$ is the damping coefficient; $\mathrm{M}$ is the transposed matrix with a positive real characteristic value that can't be irreducible, the matrix A is known as a Markov chain ${ }^{[12]}$.

\subsubsection{DWNR algorithm process}

The DWNR matrix A can be easily obtained by using formulas(6 and 9):

$$
A=\alpha * M+\frac{1-\alpha}{N} * E^{T}=\alpha * M+\frac{1-\alpha}{N} * e * e^{T}
$$

Where $\mathrm{M}$ is the transposed matrix; $\alpha$ is the damping coefficient; $\mathrm{N}$ is the sum of nodes; $E^{T}=e * e^{T}$ is the determinant of " 1 ".

The iteration process of the DWNR value is shown in Fig.2

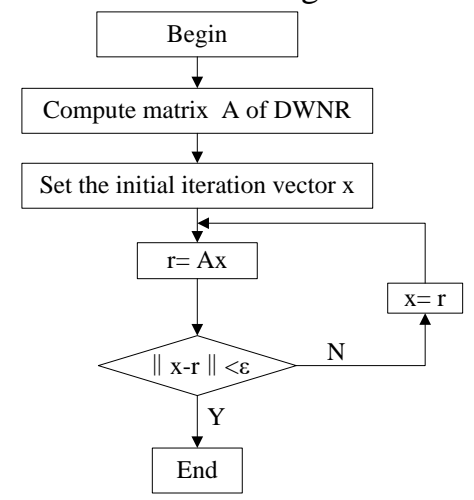

Fig.2 DWNR algorithm process

Steps are described as below:

1)Compute matrix A of DWNR;

2) Set the initial iteration vector $x$. If the DWNR value of the initial node is $x_{i}\left(x_{i}\right.$ is the DWNR initial value of node $\left.v_{i}\right)$, then $x^{T}=\left(x_{0}, x_{1}, x_{2}, \cdots, x_{n}\right)$;

3) Iterative calculation $r=A x$;

4) if $(\|x-r\|<\varepsilon)$, DWNR value of the node can be proved to satisfy the precision requirement. End the iteration and then output the result $r$, where $\varepsilon$ is the accuracy value set in advance, probably $\varepsilon=l e-8$;

5) else $x=r$, Return to step 2, go on the iteration cycle. 


\section{Simulation and Analysis}

In order to verify the effectiveness of the DWNR algorithm for the complex network, this paper simulated on the Windows XP platform with MATLAB 7.12.0. The network model has 6 nodes, and the connection relationship is shown in Fig.3

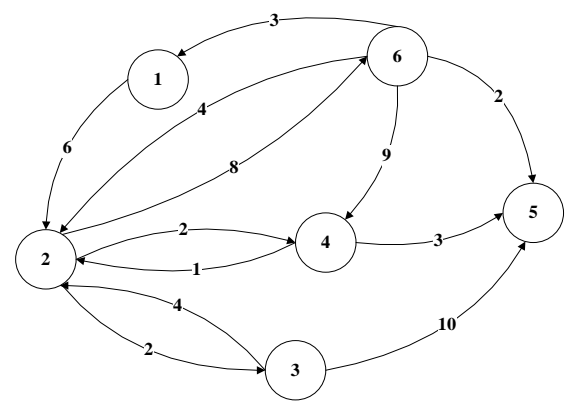

Fig.3 Connection relationship of directed weighted network

According to the above network, the matrix $\mathrm{H}, \mathrm{P}, \mathrm{P}^{\prime}$ and $\mathrm{M}$ are listed as below:

$$
H=\left(\begin{array}{llllll}
0 & 6 & 0 & 0 & 0 & 0 \\
0 & 0 & 2 & 2 & 0 & 8 \\
0 & 4 & 0 & 0 & 10 & 0 \\
0 & 1 & 0 & 0 & 3 & 0 \\
0 & 0 & 0 & 0 & 0 & 0 \\
3 & 4 & 0 & 9 & 2 & 0
\end{array}\right) \quad P=\left(\begin{array}{cccccc}
0 & 1 & 0 & 0 & 0 & 0 \\
0 & 0 & 1 / 6 & 1 / 6 & 0 & 2 / 3 \\
0 & 2 / 7 & 0 & 0 & 5 / 7 & 0 \\
0 & 1 / 4 & 0 & 0 & 3 / 4 & 0 \\
0 & 0 & 0 & 0 & 0 & 0 \\
1 / 6 & 2 / 9 & 0 & 1 / 2 & 1 / 9 & 0
\end{array}\right) \quad P^{\prime}=\left(\begin{array}{cccccc}
0 & 1 & 0 & 0 & 0 & 0 \\
0 & 0 & 1 / 6 & 1 / 6 & 0 & 2 / 3 \\
0 & 2 / 7 & 0 & 0 & 5 / 7 & 0 \\
0 & 1 / 4 & 0 & 0 & 3 / 4 & 0 \\
1 / 6 & 1 / 6 & 1 / 6 & 1 / 6 & 1 / 6 & 1 / 6 \\
1 / 6 & 2 / 9 & 0 & 1 / 2 & 1 / 9 & 0
\end{array}\right) \quad M=\left(\begin{array}{cccccc}
0 & 0 & 0 & 0 & 1 / 6 & 1 / 6 \\
1 & 0 & 2 / 7 & 1 / 4 & 1 / 6 & 2 / 9 \\
0 & 1 / 6 & 0 & 0 & 1 / 6 & 0 \\
0 & 1 / 6 & 0 & 0 & 1 / 6 & 1 / 2 \\
0 & 0 & 5 / 7 & 3 / 4 & 1 / 6 & 1 / 9 \\
0 & 2 / 3 & 0 & 0 & 1 / 6 & 0
\end{array}\right)
$$

Suppose $\alpha=0.85$, the matrix A can be easily obtained by formula(9):

$$
A=\alpha \times M+(1-\alpha) \times \frac{1}{n} e e^{T}=\left(\begin{array}{cccccc}
0.025 & 0.025 & 0.025 & 0.025 & 0.1667 & 0.1667 \\
0.875 & 0.025 & 0.2679 & 0.2375 & 0.1667 & 0.2139 \\
0.025 & 0.1667 & 0.025 & 0.025 & 0.1667 & 0.025 \\
0.025 & 0.1667 & 0.025 & 0.025 & 0.1667 & 0.445 \\
0.025 & 0.025 & 0.6321 & 0.6625 & 0.1667 & 0.1194 \\
0.025 & 0.5917 & 0.025 & 0.025 & 0.1667 & 0.025
\end{array}\right)
$$

Compute the in-strength of each node in Fig.3, and normalized to get the initial DWNR vector:

$x=\left(\begin{array}{llllll}0.0556 & 0.2778 & 0.0370 & 0.2037 & 0.2778 & 0.1481\end{array}\right)^{T}$

Compute the value of DWNR by using an iterative method.

The first iterative step:

As $\|x-r\|=0.1476>$ le -8 , continue.

$$
r=A x=A \times\left(\begin{array}{l}
0.0556 \\
0.2778 \\
0.0370 \\
0.2037 \\
0.2778 \\
0.1481
\end{array}\right)=\left(\begin{array}{l}
0.0854 \\
0.1919 \\
0.1037 \\
0.1659 \\
0.2307 \\
0.2218
\end{array}\right)
$$

Make $x=r$, The second iterative step:

$$
r=A x=A \times\left(\begin{array}{l}
0.0854 \\
0.1919 \\
0.1037 \\
0.1659 \\
0.2307 \\
0.2218
\end{array}\right)=\left(\begin{array}{l}
0.0891 \\
0.2326 \\
0.0849 \\
0.1780 \\
0.2474 \\
0.1664
\end{array}\right)
$$

As $\|x-r\|=0.0743>l e-8$, continue.

After 12,171 iterations, $\|x-r\|=9.9959 E-9<l e-8$ satisfy the conditions, end.

$3 \sim 12171$ iterations $r$ of are shown as follow:

$$
\left(\begin{array}{l}
0.0862 \\
0.2235 \\
0.0910 \\
0.1716 \\
0.2374 \\
0.1869
\end{array}\right)\left(\begin{array}{l}
0.0850 \\
0.2257 \\
0.0902 \\
0.1687 \\
0.2408 \\
0.1852
\end{array}\right)\left(\begin{array}{l}
0.0853 \\
0.2257 \\
0.0902 \\
0.1687 \\
0.2408 \\
0.1852
\end{array}\right) \cdots\left(\begin{array}{l}
0.0857 \\
0.2257 \\
0.0911 \\
0.1698 \\
0.2405 \\
0.1871
\end{array}\right)\left(\begin{array}{l}
0.0857 \\
0.2257 \\
0.0911 \\
0.1698 \\
0.2405 \\
0.1871
\end{array}\right)
$$


Parameters and Importance ranking of the nodes are shown as Fig.4

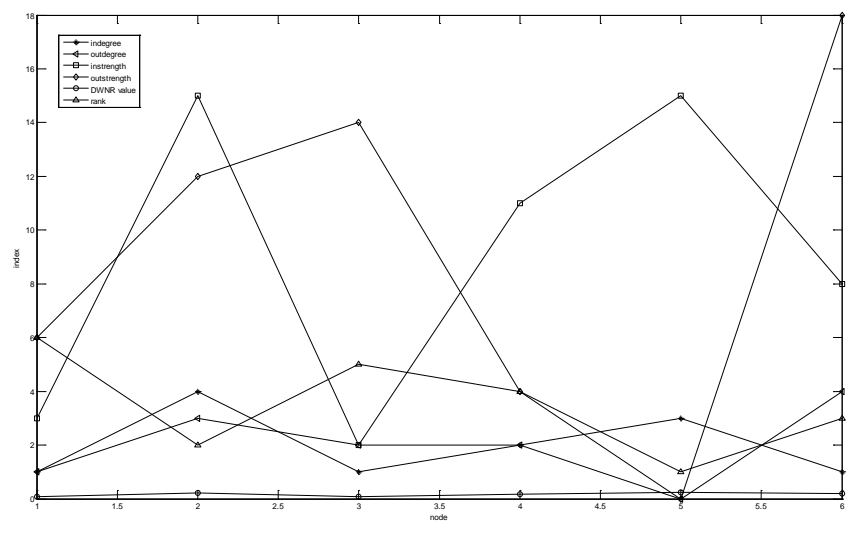

Fig.4 Simulation results

From the chart above, we can know that the importance of nodes in the directed weighted network not only relates to the number of the link source, but also relates to the importance and in-strength of the source nodes. The more in-nodes with, the more important the nodes are, and the edge is more valuable.

\section{Conclusion}

In view of the current use of complex network, this paper built an importance evaluation model based on DWNR algorithm. By analyzing the simulation, this method solves the problems that traditional method have, can be used to evaluate and rank the importance of the complex networks. The conclusion can also has a reference significance in the energy, power, communication, traffic or social networks.

\section{References}

[1] Chen Y, Hu A Q, Hu J, et al. A method for finding the most vital node in communication networks[J].High Technology Letters,2004,1 (2):573-575.

[2] Erdos P, Renyi A. On the evolution of random graphs[J].Publication of the mathematical Institute of the Hungarian Academy of Sciences,1960,6 (5):17-61.

[3] Watts D J, Strogatz S H. Collective dynamics of small world networks[J].Nature,1998,6 (393):440-442.

[4] Barabasi A L, Albert R. Emergence of scaling in random networks[J].Science,1999,6 (286):509-512.

[5] Yu Xin, Li Yanhe, Zheng Xiaoping,etc. Node importance evaluation based on communication network performance grads[J].Department of Electronic Engineering, Tsinghua University,2008,48(4):541-544.

[6] Jiang Yu, Hu Aiqun, Pan Tingting. A new method for the evaluation of the importance of communication network node-the node isolation method[J]. High-tech communication,2008,18(7): 673-678.

[7] Liu Jianqiang, Lan Julong, Wu Jiangxing. Evaluating the vital network nodes based on node estranging[J].Computer Engineering \& Science,2011,33(3):13-17.

[8] Barrat A, Barthelemy M, Vespignani A. Modeling the evolution of weighted networks [J]. Physical Review E, 2004, 70(6):1-13.

[9] Wang G Y, Zhou J, Xie Y. Directed weighted network model based on BBV[J]. Computer Networks, 2010, 36(12): 141-143.

[10] Brin S, Page. The anatomy of a large scale hyper-textual web search engine[J]. Computer Networks, 1998,30(2):107-117.

[11] Li Qingyang, Wang Nengchao, Yi Dayi. Numerical Analysis[M].Beijing: Tsinghua University Press, 2003: 299-301.

[12] Wang Zhigang, Gao Zetu, Zhang Bingxia,etc. Application of stochastic processes[M].Hefei: University of Science and Technology of China Press,2009:52-71. 